\title{
MAPK/P53-mediated FASN expression in bone tumors
}

\author{
YE JIANG ${ }^{*}$, XIAOFAN YIN*, LIANG WU*, QIANG QIN and JUN XU \\ Department of Orthopaedics, Minhang Hospital, Fudan University, Shanghai 201199, P.R. China
}

Received September 6, 2016; Accepted January 11, 2017

DOI: $10.3892 / 01.2017 .6015$

\begin{abstract}
The correlation between mitogen-activated protein kinase (MAPK)/P53 signaling-dependent fatty acid synthase (FASN) expression and bone tumors was examined in the present study. We established the SH081 bone tumor cell line, which was used to determine the expression of FASN and MAPK/P53 at the mRNA and protein levels in bone tumor cells and normal cells. Compared with the normal cells, the expression of MAPK/P53 and FASN mRNA was significantly elevated, whereas inhibition of MAPK/P53 decreased FASN expression. Similarly, the expression of FASN and MAPK/P53 proteins were significantly elevated in the bone tumor cells and treatment with the MAPK/P53 inhibitor decreased the expression of FASN. To determine the role of MAPK/P53 in cell proliferation, bone cancer cells were treated with MAPK/P53 inhibitor and the results showed a reduced proliferation rate. Thus, FASN promotes the development of bone tumors and MAPK/P53 signaling contributes to bone tumors by upregulating the expression of FASN.
\end{abstract}

\section{Introduction}

Clinical statistics show that the bone is often involved in tumor metastasis during the late stages of most tumors (1). For example, the tumor cells of breast cancer, colon cancer, and lung cancer can be found in bone tissue (2). Clinical statistics also show that the osseous tumor metastasis would cause clinical symptoms (3) such as osteoporosis and pathological fracture by influencing sclerotin and joints (4). The incidence of osteosarcoma covers $45.3 \%$ of all types of bone tumors, and is the main morbidity among teenagers (5). Although research on bone tumors has made some progress, its nosogenesis remains to be elucidated, although its occurrence is influenced by genetic and environmental factors, such as nutrition, and exercise (6).

Correspondence to: Dr Jun Xu, Department of Orthopaedics, Minhang Hospital, Fudan University, 170 Xinsong Road, Shanghai 201199, P.R. China

E-mail: grzd_938@163.com

*Contributed equally

Key words: mitogen-activated protein kinase/P53, signal pathway, fatty acid synthase, bone tumor, regulation
Previous findings showed that fatty acid synthase (FASN) plays an important role in fatty acid synthesis and its inhibition prevents the proliferation of malignant cells and promotes apoptosis (7). Moreover, tumors express high amounts of acid such as fatty acid, suggesting a key role of FASN in cancer cell metabolism. In addition, the mitogen-activated protein kinase (MAPK)/P53 signaling pathway is activated in many tumors (8). For example, the expression level of MAPK/P53 is significantly increased in tumor cells from breast and colon cancer. The MAPK/P53 signaling pathway regulates the expression of FASN in breast cancer cells to control cell proliferation (9). However, the role of FASN and MAPK/P53 signaling in bone tumor and the functional relationship of these molecules are currently unknown.

The aim of the present study was to determine the correlation between FASN and MAPK/P53 signaling and the incidence of bone tumors. The aim was to provide theoretical and experimental support to understand the role of these molecules in the pathogenesis of bone tumors.

\section{Materials and methods}

Cell culture and treatment. The SH081 bone tumor cell line was established and maintained in our laboratory. We cultured the $\mathrm{SH} 081$ cell line at $3{ }^{\circ} \mathrm{C}, 5 \% \mathrm{CO}_{2}$, and collected the cells at $90 \%$ confluence. The cells were centrifuged for $10 \mathrm{~min}$ at $1,500 \mathrm{x} \mathrm{g}$ at $4^{\circ} \mathrm{C}$, and then the fluid was added and the cells were preserved at $-80^{\circ} \mathrm{C}$. MAPK/P53 signaling pathway (100 $\mu \mathrm{l}, 100 \mathrm{ml})$ blocker (Calbiochem, Billerica, MA, USA) was added in observation group 2 during culture, and the cells were cultured at $37^{\circ} \mathrm{C}, 5 \% \mathrm{CO}_{2}$. Other materials used were: dimethyl sulphoxide, fetal bovine serum and trypsin.

$R T-q P C R$. RNA extraction: We cultured the bone tumor cells at $37^{\circ} \mathrm{C}, 5 \% \mathrm{CO}_{2}$, extracted total RNA and measured the amount of RNA (2). For qPCR the expression of MAPK/P53 and FASN mRNA in cells was analyzed. This study generated cDNA by reverse transcription of RNA as template for qPCR (Applied Biosystems Life Technologies, Foster City, CA, USA). The primer sequences are shown in Table I. Other materials used were: Transilluminator (Bio-Rad, Munchen, Germany) and high speed and low temperature refrigerated centrifuge (Hitachi Ltd., Tokyo, Japan).

Enzyme-linked immunosorbent assay (ELISA) response. We followed the manufacturer's recommendation using the ELISA kit (Takara Bio, Dalian, China) with minor modifications (10). 
Table I. qPCR primers.

\begin{tabular}{ll}
\hline Primers & \multicolumn{1}{c}{ Primer sequences } \\
\hline MAPK/P53-F & GTCGATCGTCGATCGCTACGC \\
MAPK/P53-R & CGTAGCTAGTCGATCGACTAGC \\
FASN-F & TGCTAGCTGATCGATCGATCGTCG \\
FASN-R & CGTAGCTGATCGATGCTAGCTAGC \\
GAPDH-F & TGCTAGGCTAGGACGCTAGCTAC \\
GAPDH-R & CTGGGCTAGATCGACGAGAGCTC \\
\hline
\end{tabular}

MAPK, mitogen-activated protein kinase; FASN, fatty acid synthase; F, forward; R, reverse.

The standard protein sample was diluted in assay buffer at 1:50 and the standard curve was made according to the instruction. The samples were diluted in PBS (pH 7.2) at 1:100. We added $100 \mu 1$ of the sample mix in each well, and added $50 \mu \mathrm{l}$ of detection solution. TMB chromogenic substrate was used after the sample was incubated at room temperature for $2 \mathrm{~h}$. Light absorption was measured at $495 \mathrm{~nm}$, and the concentration of MAPK/P53 and FASN in each sample was calculated according to the standard curve.

Western blot analysis. The expression of APC in different samples was detected by western blot analysis. Total protein extracted with the animal cell total protein extraction kit was performed according to the 'Guide of Molecular Clone', 3rd edition. The primary antibody used was diluted at 1:800 and the secondary antibody was diluted at 1:500. Monoclonal and polyclonal, and animal origin are referred to a previous study (2).

Detection of cell proliferation by MTT. The procedure was performed as previously described $(10,11)$.

Statistical analysis. Data were processed and analyzed by SPSS 20.0 (Chicago, IL, USA). When the test level was $\alpha=0.05$, the difference was significant at $\mathrm{P}<0.05$. When test level was $\alpha=0.01$, the difference was significant at $\mathrm{P}<0.01$.

\section{Results}

MAPK/P53 and FASN mRNA expression in normal and bone tumor cells. Using SH081 bone tumor cells and normal cells, total RNA was extracted to determine the expression levels of MAPK/P53 and FASN mRNA by qPCR. The expression level of MAPK/P53 mRNA in bone tumor cells was 8.74-fold higher than that in the normal cells, and the difference was statistically significant (Fig. 1). The expression levels of FASN mRNA in bone tumor cells was 13.3 times higher compared to normal cells, and statistically significant (Fig. 1). This result indicated robust upregulation of MAPK/P53 and FASN in bone tumors.

MAPK/P53 and FASN mRNA expression following $M A P K / P 53$ inhibition. We then examined the consequence of inhibiting MAPK/P53 in bone tumor cells. MAPK/P53 and

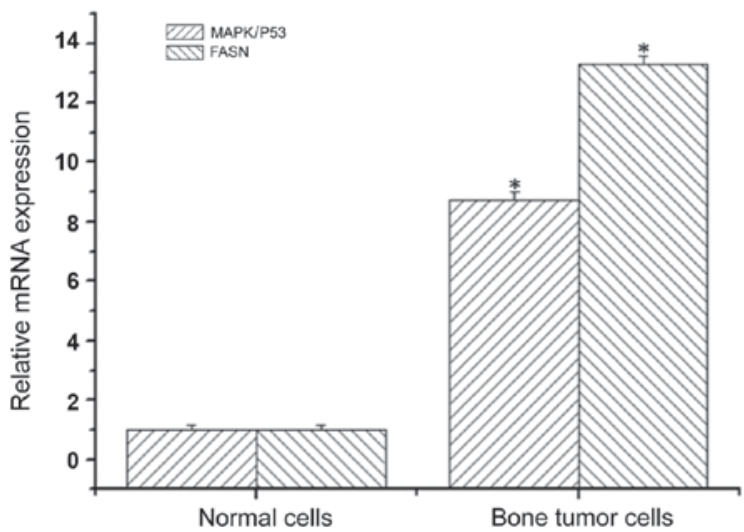

Figure 1. Expression levels of MAPK/P53 and FASN mRNA in normal cells and SH081 bone tumor cells. Asterisk indicates significant difference between groups $(\mathrm{P}<0.05)$. MAPK, mitogen-activated protein kinase; FASN, fatty acid synthase.

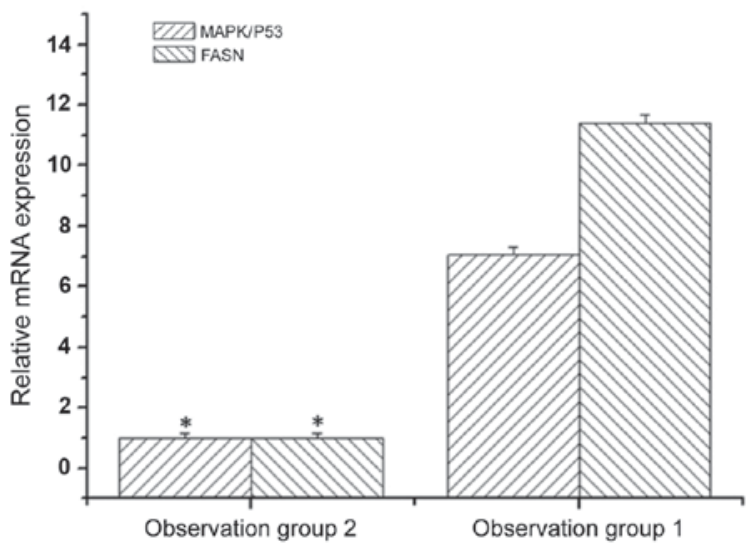

Figure 2. Expression levels of MAPK/P53 and FASN mRNA in SH081 bone tumor cells treated with MAPK/P53 inhibitor. Asterisk indicates significant difference between groups $(\mathrm{P}<0.05)$. MAPK, mitogen-activated protein kinase; FASN, fatty acid synthase.

FASN mRNA levels were analyzed in treated and non-treated SH081 bone tumor cells by qPCR. The expression level of MAPK/P53 mRNA in the non-treated bone tumor cells was 7.05-fold higher than the level in bone tumor cells treated with MAPK/P53 inhibitor (Fig. 2). The expression levels of FASN mRNA in the non-treated cells was 11.4-fold higher compared to the SH081 cells treated with MAPK/P53 inhibitor (Fig. 2). These results indicated that MAPK/P53 regulates the expression of FASN gene in bone tumor cells.

Determination of MAPK/P53 and FASN expression using western blot analysis. To verify the changes in mRNA, we extracted total protein extracted from normal cells and SH081 bone tumor cells and measured the expression of the MAPK/P53 and FASN proteins by western blot analysis. The expression levels of MAPK/P53 and FASN proteins in normal cells were significantly lower than in the bone tumor cells (Figs. 3 and 4). This result was in accordance with the results observed by qPCR.

Expression of MAPK/P53 and FASN by ELISA. To verify the changes in MAPK/P53 and FASN proteins by a more 


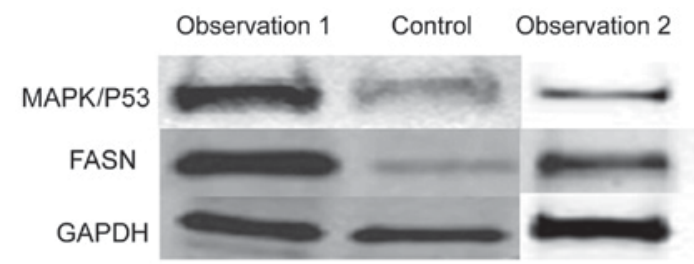

Figure 3. Expression levels of MAPK/P53 and FASN proteins in normal cells and SH081 bone tumor cells by western blot analysis. MAPK, mitogen-activated protein kinase; FASN, fatty acid synthase.

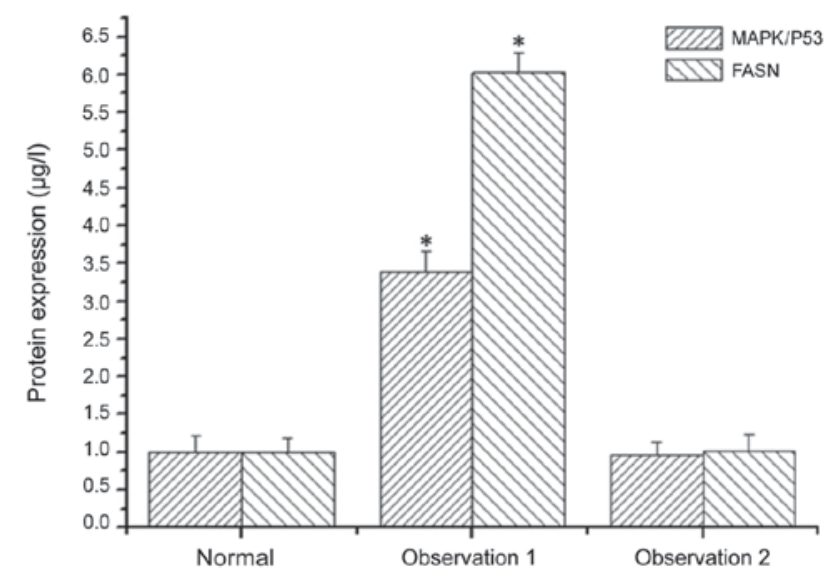

Figure 4. Quantification of MAPK/P53 and FASN proteins in normal cells and $\mathrm{SH} 081$ bone tumor cells by western blot analysis. Asterisk indicates significant difference between groups. MAPK, mitogen-activated protein kinase; FASN, fatty acid synthase.

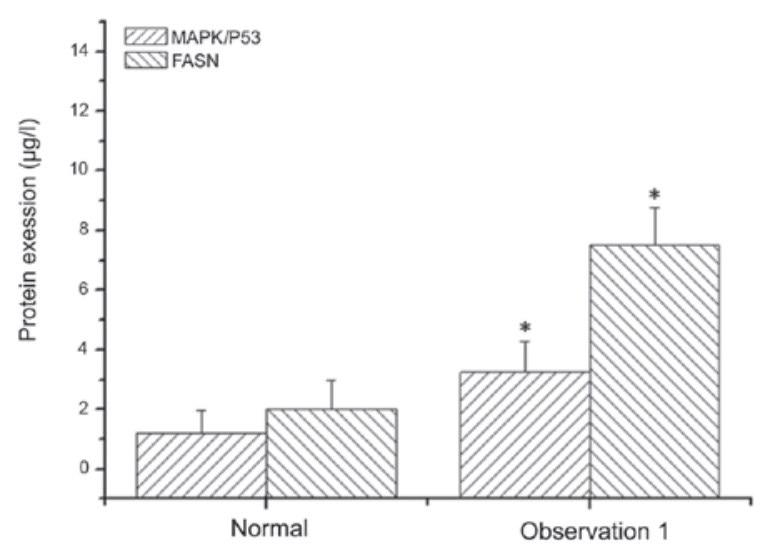

Figure 5. Expression levels of MAPK/P53 and FASN proteins in normal cells and SH081 bone tumor cells. Asterisk indicates significant difference between groups. MAPK, mitogen-activated protein kinase; FASN, fatty acid synthase.

quantitative technique, we measured the expression by ELISA. The expression level of MAPK/P53 in SH081 bone tumor cells was $3.25 \pm 1.03 \mu \mathrm{l} / \mathrm{l}$, but significantly higher in normal cells $(1.21 \pm 0.76 \mu \mathrm{l} / \mathrm{l})$ (Fig. 5). The expression level FASN in normal cells was $2.03 \pm 0.96 \mu \mathrm{l} / 1$, which was significantly lower than in bone tumor cells (7.52 $\pm 1.25 \mu 1 / 1)$ (Fig. 5). inhibition by ELISA. The expression levels of MAPK/P53 and

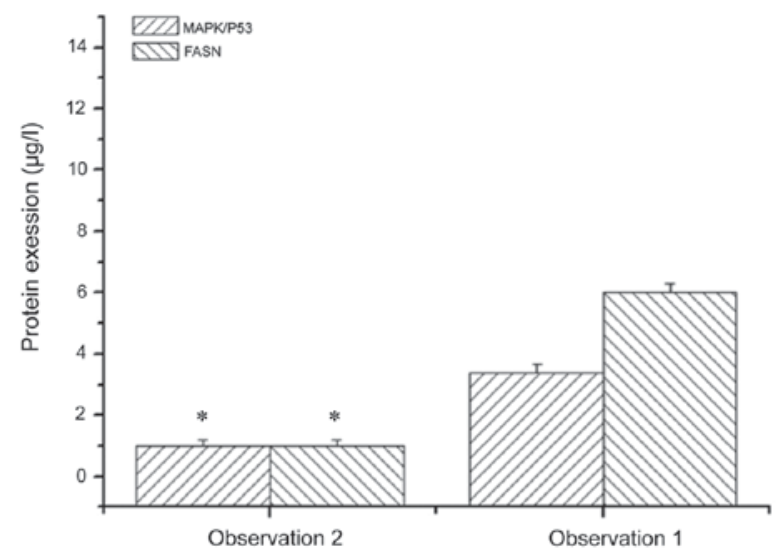

Figure 6. The expression levels of the protein of MAPK/P53 and FASN in SH081 bone tumor cells treated with MAPK/P53 inhibitor. Asterisk indicates significant difference between groups. MAPK, mitogen-activated protein kinase; FASN, fatty acid synthase.

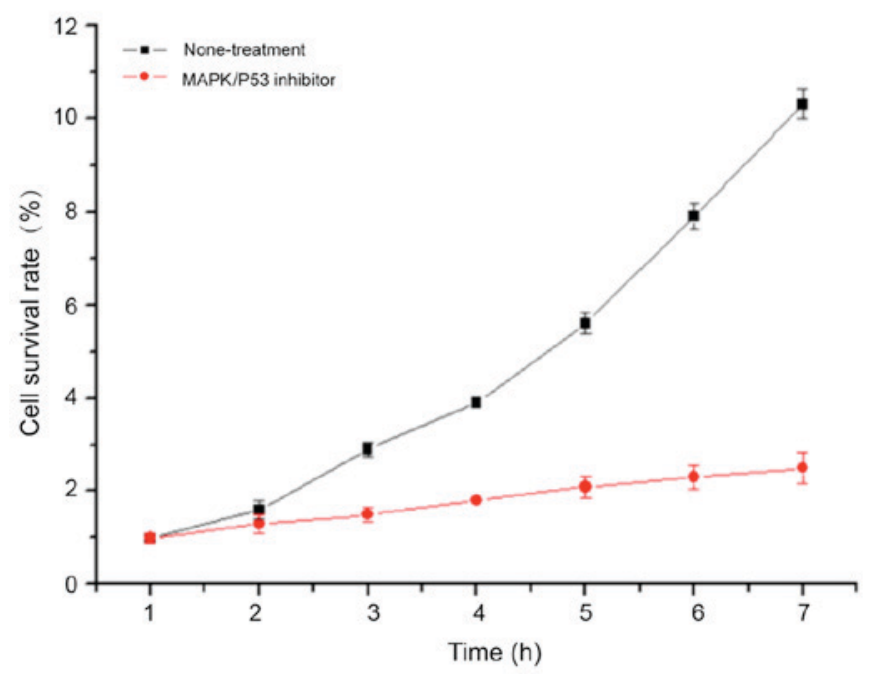

Figure 7. Cell proliferation in SH081 bone tumor cells treated with MAPK/P53 inhibitor by MTT method. MAPK, mitogen-activated protein kinase.

FASN in SH081 bone tumor cells treated and not treated with MAPK/P53 inhibitor were examined. The expression level of MAPK/P53 in bone tumor cells not treated with MAPK/P53 inhibitor was 3.39-fold higher than bone tumor cells treated with MAPK/P53 inhibitor (Fig. 6). The expression levels of FASN in bone tumor cells not treated with MAPK/P53 inhibitor was 6.03-fold higher than in bone tumor cells treated with MAPK/P53 inhibitor (Fig. 6). These results indicated that MAPK/P53 regulates the expression of FASN in bone tumor cells, which was consistent with the qPCR and western blot results.

Proliferation of bone tumor cells treated with MAPK/P53 inhibitor. The cell proliferation of bone tumor cells treated and not treated with MAPK/P53 inhibitor was assessed to determine the functional relevance of the activation of the MAPK/P53 signaling pathway. Compared with the SH081 cells not treated with MAPK/P53 inhibitor, the cell proliferation of bone tumor cells treated with MAPK/P53 inhibitor was 
significantly reduced (Fig. 7). This result suggested that the MAPK/P53 signaling pathway promotes the proliferation of bone tumor cells.

\section{Discussion}

FASN in cells mainly participates in the metabolic process of acidic materials such as fatty acid (12). Moreover, FASN has various enzymatic activities such as condensation, dehydration, and polymerization in plants. Previously, it has been found that the $\mathrm{pH}$ of the bone tumor fluid was significantly lower than that in normal tissues. Analysis of the main constituents of bone tumor fluids shows that the content of acidic materials such as fatty acid is elevated, suggesting that in bone tumor the metabolism and synthesis of fatty acids and other acidic materials undergo pathological changes. The expression level of FASN in tumor cells such as breast and bladder cancer cells was apparently higher than that in normal cells (13). Breast cancer cells treated with FASN inhibitor exhibit a lower rate of proliferation and a higher ratio of apoptosis, which suggests that FASN regulates proliferation and apoptosis in breast cancer. Further research shows that in colon cancer cells FASN inhibits cell growth cycle transition from $\mathrm{S}$ to $\mathrm{G} 2 / \mathrm{M}$ stage (10), providing theoretical support for FASN inhibition of cancer cell proliferation.

The MAPK signaling pathway is a main signal transduction pathway closely connected with several stress reactions, and physical and chemical reactions within the cells. For example, MAPK signaling was involved in the cell response to outside stimuli by activating and regulating the client protein, such as P38MAPK (14). Additionally, the expression level of MAPK in tumor cells was apparently higher than that in normal cells $(15,16)$. Furthermore, inhibition of MAPK signaling in breast cancer impairs proliferation and promotes apoptosis (17). To the best of our knowledge, in the present study, we have shown for the first time that the expression level of FASN was significantly elevated in bone tumor cells compared to normal cells with the expression level of MAPK/P53. The expression level of FASN in tumor cells treated with MAPK/P53 inhibitor significantly decreased, and the proliferation rate was reduced. This indicates that FASN participates in bone tumor proliferation by regulating MAPK/P53 activity, and as a result participate in the occurrence and progress of bone tumors.

\section{References}

1. Zhao Q, Liu ZD, Xue Y, Wang JF, Li H, Tang QJ, Wang YM, Dong P and Xue CH: Ds-echinoside A, a new triterpene glycoside derived from sea cucumber, exhibits antimetastatic activity via the inhibition of NF- $\mathrm{kB}$-dependent MMP-9 and VEGF expressions. J Zhejiang Univ Sci B 12: 534-544, 2011
2. LiN,BuX,WuP,WuPand Huang P: The 'HER2-PI3K/Akt-FASN Axis' regulated malignant phenotype of colorectal cancer cells. Lipids 47: 403-411, 2012.

3. Toegel S, Wu SQ, Otero M, Goldring MB, Leelapornpisid P, Chiari C, Kolb A, Unger FM, Windhager R and Viernstein H: Caesalpinia sappan extract inhibits IL1 $\beta$-mediated overexpression of matrix metalloproteinases in human chondrocytes. Genes Nutr 7: 307-318, 2012.

4. Xie X, Li W, Lan T, Liu W, Peng J, Huang K, Huang J, Shen X, Liu $\mathrm{P}$ and Huang $\mathrm{H}$ : Berberine ameliorates hyperglycemia in alloxan-induced diabetic C57BL/6 mice through activation of Akt signaling pathway. Endocr J 58: 761-768, 2011.

5. Liu W, Zhang X, Liu P, Shen X, Lan T, Li W, Jiang Q, Xie X and Huang $\mathrm{H}$ : Effects of berberine on matrix accumulation and NF-kappa B signal pathway in alloxan-induced diabetic mice with renal injury. Eur J Pharmacol 638: 150-155, 2010.

6. Yu S, Yu Y, Liu L, Wang X, Lu S, Liang Y, Liu X, Xie L and Wang G: Increased plasma exposures of five protoberberine alkaloids from Coptidis Rhizoma in streptozotocin-induced diabetic rats: is P-GP involved? Planta Med 76: 876-881, 2010.

7. Tahara A, Tsukada J, Tomura Y, Yatsu T and Shibasaki M: Effects of high glucose on AVP-induced hyperplasia, hypertrophy, and type IV collagen synthesis in cultured rat mesangial cells. Endocr Res 37: 216-227, 2012.

8. Ponchiardi C, Mauer M and Najafian B: Temporal profile of diabetic nephropathy pathologic changes. Curr Diab Rep 13: 592-599, 2013.

9. Lv ZM, Wang Q, Wan Q, Lin JG, Hu MS, Liu YX and Wang R: The role of the p38 MAPK signaling pathway in high glucose-induced epithelial-mesenchymal transition of cultured human renal tubular epithelial cells. PLoS One 6: e22806, 2011.

10. Oh D, Lee Y, La B, Yeo J, Chung E, Kim Y and Lee C: Fatty acid composition of beef is associated with exonic nucleotide variants of the gene encoding FASN. Mol Biol Rep 39: 4083-4090, 2012.

11. de Souza FR, Chiquitelli MG, da Fonseca LF, Cardoso DF, da Silva Fonseca PD, de Camargo GM, Gil FM, Boligon AA, Tonhati $\mathrm{H}$, Mercadante ME, et al: Associations of FASN gene polymorphisms with economical traits in Nellore cattle (Bos primigenius indicus). Mol Biol Rep 39: 10097-10104, 2012.

12. Yeon SH, Lee SH, Choi BH, Lee HJ, Jang GW, Lee KT, Kim KH, Lee JH and Chung HY: Genetic variation of FASN is associated with fatty acid composition of Hanwoo. Meat Sci 94: 133-138, 2013.

13. Alim MA, Wang P, Wu XP, Li C, Cui XG, Zhang SL, Zhang Q, Zhang Y and Sun DX: Effect of FASN gene on milk yield and milk composition in the Chinese Holstein dairy population. Anim Genet 45: 111-113, 2014.

14. Zhu JJ, Luo J, Wang W, Yu K, Wang HB, Shi HB, Sun YT, Lin XZ and Li J: Inhibition of FASN reduces the synthesis of medium-chain fatty acids in goat mammary gland. Animal 8: 1469-1478, 2014

15. Mohammadi H, Shahrebabak MM and Sadeghi M: Association between single nucleotide polymorphism in the ovine DGAT1 gene and carcass traits in two Iranian sheep breeds. Anim Biotechnol 24: 159-167, 2013.

16. Nafikov RA, Schoonmaker JP, Korn KT, Noack K, Garrick DJ, Koehler KJ, Minick-Bormann J, Reecy JM, Spurlock DE and Beitz DC: Sterol regulatory element binding transcription factor 1 (SREBF1) polymorphism and milk fatty acid composition. J Dairy Sci 96: 2605-2616, 2013.

17. Jin YC, Li ZH, Hong ZS, Xu CX, Han JA, Choi SH, Yin JL, Zhang QK, Lee KB, Kang SK, et al: Conjugated linoleic acid synthesis-related protein proteasome subunit $\alpha 5$ (PSMA5) is increased by vaccenic acid treatment in goat mammary tissue. J Dairy Sci 95: 4286-4297, 2012. 